\title{
Accuracy of Mean Field Approximations for Atoms and Molecules
}

\section{Volker Bach}

Fachbereich Mathematik MA 7-2, Technische Universität Berlin, Straße des 17. Juni 136, D-10623 Berlin, Germany

Received July 9, 1992; in revised form November 11, 1992

Abstract. We estimate the accuracy of the mean field approximation induced by the Thomas-Fermi potential for the ground state energy of atoms and molecules. Taking the Dirac exchange correction into account, we show the error to be of the form $O\left(Z^{5 / 3-\delta}\right)+D$ for any $\delta<2 / 231$ as the total nuclear charge $Z$ becomes large. $D$ is an electrostatic energy of the difference density that measures the deviation of the mean field ground state from self-consistency.

\section{Introduction}

The nonrelativistic quantum mechanical model for an atom $(K=1)$ or molecule is given by the Hamiltonian

$$
H_{N}(\underline{Z}, \underline{R}):=\sum_{i=1}^{N}\left(-\Delta_{i}-\sum_{j=1}^{K} \frac{Z_{j}}{\left|x_{i}-R_{j}\right|}\right)+\sum_{1 \leqq i<j}^{N} \frac{1}{\left|x_{i}-x_{j}\right|},
$$

acting as a self-adjoint operator on $D_{N}:=\bigwedge_{i=1}^{N} \tilde{\mathscr{H}} \subseteq \mathscr{H}_{N}:=\bigwedge_{i=1}^{N} \mathscr{H}$, $\mathscr{H}:=L^{2}\left(\mathbb{R}^{3}\right) \otimes \mathbb{C}^{m}, \tilde{\mathscr{H}}:=H^{2}\left(\mathbb{R}^{3}\right) \otimes \mathbb{C}^{m}$. Here, $\underline{Z}:=\left(Z_{1}, \ldots, Z_{K}\right)$ and $\underline{R}:=$ $\left(R_{1}, \ldots, R_{K}\right)$ denote the charges and positions of the nuclei. We will drop this dependence in our notation henceforth. Unless stated otherwise, the operators are always assumed to act as identity on $\mathbb{C}^{m}$.

We are interested in approximations for the ground state energy

$$
E_{Q}(N):=\inf \left\{\left\langle\Psi_{N}\left|H_{N}\right| \Psi_{N}\right\rangle \mid \Psi_{N} \in D_{N},\left\|\Psi_{N}\right\|=1\right\} .
$$

The most widely used one in physics is the Mean Field approximation. It consists in replacing the pair potential $\sum_{1 \leqq i<j}^{N} \frac{1}{\left|x_{i}-x_{j}\right|}$ in (1) by an average one-body potential

$$
\sum_{i=1}^{N} \int \frac{d^{3} y}{\left|x_{i}-y\right|} \rho(y)-\frac{1}{2} D(\rho, \rho)
$$


where $D(f, g):=\int \frac{d^{3} x d^{3} y}{|x-y|} \overline{f(x)} g(y)$ induces the Coulomb norm and $\frac{1}{2} D(\rho, \rho)$ is the electrostatic energy of a nonnegative density $\rho \in L^{1}\left(\mathbb{R}^{3}\right)$. So, provided $D(\rho, \rho)<\infty$, the substitute Hamiltonian reads

$$
H_{N}(\rho):=\sum_{i=1}^{N}\left(-\Delta_{i}-\sum_{j=1}^{K} \frac{Z_{j}}{\left|x_{i}-R_{j}\right|}+\int \frac{d^{3} y}{\left|x_{i}-y\right|} \rho(y)\right)-\frac{1}{2} D(\rho, \rho),
$$

and is defined on $D_{N}$. In the case of atoms and molecules with $N$ electrons a natural candidate for $\rho$ is the corresponding Thomas-Fermi (TF) density $\rho_{\mathrm{TF}}$, and the Mean Field potential becomes the TF-potential $\phi_{\mathrm{TF}}$ (see [11]). We introduce

$$
h_{\mathrm{TF}}:=-\Delta-\sum_{j=1}^{K} \frac{Z_{j}}{\left|x-R_{j}\right|}+\int \frac{d^{3} y}{|x-y|} \rho_{\mathrm{TF}}=-\Delta-\phi_{\mathrm{TF}}(x) \text {, }
$$

self-adjointly realized on $D$. By general arguments $\sigma_{\text {ess }}\left(h_{\mathrm{TF}}\right)=[0, \infty)$ and $\sigma_{\text {disc }}\left(h_{\mathrm{TF}}\right) \subseteq(-\infty, 0)$. Moreover, if $N \geqq Z$ then the chemical potential $\mu=0$ [11] and $\left|\sigma_{\mathrm{disc}}\left(h_{\mathrm{TF}}\right)\right|<\infty$, whereas $\mu>0$ and $\left|\sigma_{\mathrm{disc}}\left(h_{\mathrm{TF}}\right)\right|=\infty$ for $N<Z$. We set $P_{N}:=\chi_{(-\infty, 0)}\left[h_{\mathrm{TF}}\right]$ in case $\operatorname{tr}_{1}\left\{\chi_{(-\infty, 0)}\left[h_{\mathrm{TF}}\right]\right\} \leqq N$. Otherwise

$$
P_{N}:=\sum_{i=1}^{N}\left|\varphi_{i}\right\rangle\left\langle\varphi_{i}\right|
$$

is a spectral projection onto $N$ eigenfunctions $\varphi_{i}$ of $h_{\mathrm{TF}}$ with lowest possible negative eigenvalue. Note that $P_{N}$ might be nonunique due to degeneracy of the $N^{\text {th }}$ eigenvalue and we pick just any of the possible ones. We observe $\operatorname{tr}_{1}\left\{P_{N}\right\} \leqq N$. For any nonnegative trace class operator $d=\sum_{i} \lambda_{i}\left|\chi_{i}\right\rangle\left\langle\chi_{i}\right|$ on $\mathscr{H}$ with orthonormal $\chi_{i}$ we call $\rho[d](x):=\sum_{\sigma=1}^{m} \sum_{i} \lambda_{i}\left|\chi_{i}(x, \sigma)\right|^{2}$ the corresponding density. In particular, $\rho_{N}:=\rho\left[P_{N}\right]$. We are now in the position to formulate our main result

Theorem 1. Consider a molecule of nuclear charges $Z_{j}$ at positions $R_{j}, 1 \leqq j \leqq K$, with fixed ratios $Z_{j} / Z$, where $Z:=\sum_{j=1}^{K} Z_{j}$ and $K \geqq 1$. Let $N \leqq Z+c Z^{1-2 / 77}$. Then for any $0<\delta<2 / 231$ there exists $c_{\delta}>0$ such that

$$
\begin{aligned}
-c_{\delta} K^{2} \cdot Z^{5 / 3-\delta} & \leqq E_{Q}(N)-\left\{\operatorname{tr}_{1}\left\{h_{\mathrm{TF}} P_{N}\right\}-\frac{1}{2} D\left(\rho_{\mathrm{TF}}, \rho_{\mathrm{TF}}\right)-c_{D} \int d^{3} x \rho_{\mathrm{TF}}^{4 / 3}(x)\right\} \\
& \leqq c_{\delta} K^{2} \cdot Z^{5 / 3-\delta}+\frac{1}{2} D\left(\rho_{N}-\rho_{\mathrm{TF}}, \rho_{N}-\rho_{\mathrm{TF}}\right)
\end{aligned}
$$

where $c_{D}:=\frac{3}{4 \pi}\left(\frac{6 \pi^{2}}{m}\right)^{1 / 3}$.

The contribution $c_{D}\left\|\rho_{\mathrm{TF}}\right\|_{4 / 3}^{4 / 3}$ in $E_{Q}(N)$ has been proposed by Dirac [2] and is due to exchange corrections. We remark that $c_{\delta}$ above is independent of $\underline{Z} / Z, K, \underline{R}$, and $N / Z$. Theorem 1 states that up to errors of $O\left(Z^{5 / 3-\delta}\right)$ the ground state energy $E_{Q}(N)$ can be evaluated by solving the eigenvalue problem for the Schrödinger operator $h_{\mathrm{TF}}$, provided this spectral analysis yields

$$
D\left(\rho_{N}-\rho_{\mathrm{TF}}, \rho_{N}-\rho_{\mathrm{TF}}\right)=O\left(Z^{5 / 3-\delta}\right) .
$$

Indeed, $D^{1 / 2}\left(\rho_{N}-\rho_{\mathrm{TF}}, \rho_{N}-\rho_{\mathrm{TF}}\right)$ is a natural norm to measure the quality of our choice $\rho=\rho_{\mathrm{TF}}$ of the Mean Field density, for it is positive definite and vanishes in case of self-consistency $\rho_{N}=\rho_{\mathrm{TF}}$. This indicates that $D^{1 / 2}\left(\rho_{N}-\rho_{\mathrm{TF}}, \rho_{N}-\rho_{\mathrm{TF}}\right)$ 
measures the deviation of the Mean Field energy

$$
E_{\mathrm{MF}}(N):=\operatorname{tr}_{1}\left\{h_{\mathrm{TF}} P_{N}\right\}-\frac{1}{2} D\left(\rho_{\mathrm{TF}}, \rho_{\mathrm{TF}}\right)-c_{D} \int d^{3} x \rho_{\mathrm{TF}}^{4 / 3}(x)
$$

from the Hartree-Fock energy $E_{\mathrm{HF}}(N)$ of the system, disregarding exchange corrections. The question how far $E_{Q}(N)$ then deviates from $E_{\mathrm{HF}}(N)$ has already been settled within the desired accuracy in [1] and we consider the present work as a continuation of [1]. Because of its importance for the present work we quote Theorem 1 from [1] which we are going to use here as a separate theorem.

Theorem 2. Fix $\underline{Z}, \underline{R}$ and let $\gamma$ be the 1-particle density matrix of a $\mu$-approximate ground state $\psi_{N} \in \bar{D}_{N}$ of $H_{N}$, i.e. $\left\langle\psi_{N} \mid H_{N} \psi_{N}\right\rangle \leqq E_{Q}(N)+\mu$. Then for any $0<\delta<1 / 12$ it holds

$$
\left|E_{Q}(N)-E_{\mathrm{HF}}(N)\right| \leqq d_{\delta} Z N^{2 / 3}\left(\frac{\operatorname{tr}_{1}\left\{\gamma-\gamma^{2}\right\}}{\mathrm{Z}}\right)^{1 / 3-\delta}+\mu,
$$

where $d_{\delta}:=828 \delta^{-1 / 3} m^{2 / 3}$.

In the atomic case, i.e. $K=1$ with $N \geqq Z, \rho_{\mathrm{TF}}$ is radially symmetric and the eigenvalue problem for $h_{\mathrm{TF}}$ is even one-dimensional. However, the desired accuracy $Z^{5 / 3-\delta}$ for $\operatorname{tr}_{1}\left\{h_{\mathrm{TF}} P_{N}\right\}$ and $D\left(\rho_{N}-\rho_{\mathrm{TF}}, \rho_{N}-\rho_{\mathrm{TF}}\right)$ requires a quite delicate WKBanalysis of $h_{\mathrm{TF}}$, which is interesting in its own right, but shall not concern us here. This analysis has been carried out by Fefferman and Seco [5] and we simply quote their claim (B) (p. 7) in [6] to supplement Theorem 1 in the atomic case: For $\rho^{\prime}:=\rho\left[\chi_{(-\infty, 0)}\left(h_{\mathrm{TF}}\right)\right]$ and some $\delta>0$ it holds

$$
D\left(\rho^{\prime}-\rho_{\mathrm{TF}}, \rho^{\prime}-\rho_{\mathrm{TF}}\right)=O\left(Z^{5 / 3-\delta}\right) .
$$

Fefferman and Seco also established Theorem 1 in [6] for atoms with $E_{Q}(N)$ replaced by $\inf _{N} E_{Q}(N)$, but their method is completely different from ours. We will prove this fact as a corollary of Theorem 1.

Corollary 1. Consider an atom, i.e. $K=1$. There exists $c_{\delta}>0$ for any $0<\delta<2 / 231$ such that

$$
\begin{aligned}
-c_{\delta} K^{2} \cdot Z^{5 / 3-\delta} & \leqq \inf _{N} E_{Q}(N)-\left\{\operatorname{tr}_{1}\left\{\left[h_{\mathrm{TF}}\right]_{-}\right\}-\frac{1}{2} D\left(\rho_{\mathrm{TF}}, \rho_{\mathrm{TF}}\right)-c_{D} \int d^{3} x \rho_{\mathrm{TF}}^{4 / 3}(x)\right\} \\
& \leqq c_{\delta} K^{2} \cdot Z^{5 / 3-\delta}+\frac{1}{2} D\left(\rho^{\prime}-\rho_{\mathrm{TF}}, \rho^{\prime}-\rho_{\mathrm{TF}}\right)
\end{aligned}
$$

where $\phi_{\mathrm{TF}}$ is the neutral TF-potential, $\rho_{\mathrm{TF}}$ is the neutral TF-density and $\rho^{\prime}:=\rho\left[\chi_{(-\infty, 0)}\left(h_{\mathrm{TF}}\right)\right]$.

This corollary follows if we set $N:=Z+Z^{1-2 / 77}$ in Theorem 1 , because it is known that $E_{Q}(N)=\inf _{N} E_{Q}(N)$ for $N \geqq Z+c Z^{1-9 / 56} \quad[4,15]$, and that $P_{N}=\chi_{(-\infty, 0)}\left[h_{\mathrm{TF}}\right]$ for $N \geqq Z+c Z^{2 / 3}[7]$.

Moreover, Fefferman and Seco compute

$$
\operatorname{tr}_{1}\left\{\left[h_{\mathrm{TF}}\right]_{-}\right\}=E_{\mathrm{TF}}(1,1) Z^{7 / 3}+\frac{m}{8} Z^{2}+\frac{2}{9} c_{D}\left\|\rho_{\mathrm{TF}}\right\|_{4 / 3}^{4 / 3}+O\left(Z^{5 / 3-\delta}\right)
$$


in the case of the neutral TF-atom. This justifies a claim of Schwinger [14] who predicted the total contribution in order $Z^{5 / 3}$ to the ground state energy of a neutral atom to be given by $\frac{11}{9} c_{D}\left\|\rho_{\mathrm{TF}}\right\|_{4 / 3}^{4 / 3}$, for in this case $\left\|\rho_{\mathrm{TF}}\right\|_{4 / 3}^{4 / 3}=c Z^{5 / 3}$ follows from scaling and $Z \leqq N \leqq Z+c Z^{2 / 3}$ is still considered "neutral."

This paper is organized as follows. In Sect. 2 we reformulate the Hartree-Fockand Mean Field approximation in terms of one-particle density matrices. In Sect. 3 we derive an abstract estimate on the error term in Theorem 1, which is estimated semiclassically in Sect. 4. For this semiclassical estimate we use a coherent state method rather than invoking a result of Ivrii and Sigal [9]. This is for two reasons; first it illustrates that leading order asymptotics suffice to make our method work. And secondly, it enables us to trace back the exchange correction to the one proposed by Dirac [2] in Sect. 5. Finally, the proof of Theorem 1 is given at the end of Sect. 5.

\section{One-Particle Density Matrices}

We set

$$
h:=-\Delta-\sum_{j=1}^{K} \frac{Z_{j}}{\left|x-R_{j}\right|}, \quad V:=\frac{1}{|x-y|},
$$

on $D:=H^{2}\left(\mathbb{R}^{3}\right) \otimes \mathbb{C}^{m} \subseteq \mathscr{H}$ and $D \otimes D$, respectively, and write

$$
H_{N}=\sum_{i=1}^{N} h_{i}+\frac{1}{2} \sum_{i \neq j} V_{i j}
$$

the indices specifying the components in $\bigotimes_{i=1}^{N} D$ the operators act on. For a normalized Slater determinant $\phi_{N}:=(N !)^{-1 / 2} \sum_{\pi}(-1)^{\pi} \chi_{\pi(1)} \otimes \cdots \otimes \chi_{\pi(N)} \in D_{N}$ we compute

$$
\left\langle\phi_{N} \mid H_{N} \phi_{N}\right\rangle=\operatorname{tr}_{1}\left\{h \gamma_{\phi}\right\}+\frac{1}{2} \operatorname{tr}_{2}\left\{V(1-E x)\left(\gamma_{\phi} \otimes \gamma_{\phi}\right)\right\}=: \varepsilon_{\mathrm{HF}}\left(\gamma_{\phi}\right)
$$

where $\gamma_{\phi}:=\sum_{i=1}^{N}\left|\chi_{i}\right\rangle\left\langle\chi_{i}\right|$ and $E x:=\sum_{i, j}\left|\varphi_{i} \otimes \varphi_{j}\right\rangle\left\langle\varphi_{j} \otimes \varphi_{i}\right|$ for any ON-basis $\left\{\varphi_{i}\right\}_{l \in \mathbb{N}}$ in $\mathscr{H}$. Conversely, a given $\gamma=\gamma^{\dagger}=\gamma^{2}, \operatorname{tr}_{1}\{\gamma\}=N$ can be associated with the normalized Slater determinant $\phi_{N}$ built from its eigenfunctions: $\gamma=\gamma_{\phi}$. The Hartree-Fock energy, i.e. the infimum of all the expectation values of the form (16), may thus be rewritten as

$$
E_{\mathrm{HF}}(N)=\inf \left\{\varepsilon_{\mathrm{HF}}(\gamma) \mid \gamma=\gamma^{\dagger}=\gamma^{2}, \operatorname{tr}_{1}\{\gamma\}=N, \operatorname{tr}_{1}\{h \gamma\}<\infty\right\} .
$$

By Lieb's variational principle $[12,1]$, we may weaken $\gamma=\gamma^{\dagger}=\gamma^{2}$ to $0 \leqq \gamma \leqq 1$ and by weak lower semicontinuity [16] $\operatorname{tr}_{1}\{\gamma\}=N$ to $\operatorname{tr}_{1}\{\gamma\} \leqq N$ in (17), so

$$
E_{\mathrm{HF}}(N)=\inf \left\{\varepsilon_{\mathrm{HF}}(\gamma) \mid 0 \leqq \gamma \leqq 1, \operatorname{tr}_{1}\{\gamma\} \leqq N, \operatorname{tr}_{1}\{h \gamma\}<\infty\right\}
$$

We rewrite the Mean Field energy in a similar fashion. Define $0 \leqq d_{\mathrm{TF}} \leqq N$ on $\mathscr{H}$ by the integral kernel $d_{\mathrm{TF}}\left(x, \sigma \mid x^{\prime}, \sigma^{\prime}\right):=q^{-1} \delta_{\sigma \sigma^{\prime}} \rho_{\mathrm{TF}}^{1 / 2}(x) \rho_{\mathrm{TF}}^{1 / 2}(y)$, so its diagonal 
summed over the spin variable equals $\rho_{\mathrm{TF}}$. Then we obtain

$$
\begin{aligned}
E_{\mathrm{MF}}(N) & =\operatorname{tr}_{1}\left\{h P_{N}\right\}+\operatorname{tr}_{2}\left\{V\left(d_{\mathrm{TF}} \otimes P_{N}\right)\right\}-\frac{1}{2} \operatorname{tr}_{2}\left\{V\left(d_{\mathrm{TF}} \otimes d_{\mathrm{TF}}\right)\right\}-c_{D} \int d^{3} x \rho_{\mathrm{TF}}^{4 / 3}(x) \\
& =: \tilde{E}_{\mathrm{MF}}(N)-\left[c_{D} \int d^{3} x \rho_{\mathrm{TF}}^{4 / 3}(x)-\frac{1}{2} \operatorname{tr}_{2}\left\{V \operatorname{Ex}\left(P_{N} \otimes P_{N}\right)\right\}\right] .
\end{aligned}
$$

This notation makes the following lemmata completely transparent.

Lemma 1. Let $0 \leqq \gamma \leqq 1, \operatorname{tr}_{1}\{\gamma\} \leqq N$ and $\operatorname{tr}_{1}\{h \gamma\}<\infty$. Then

$$
\varepsilon_{\mathrm{HF}}(\gamma) \geqq \tilde{E}_{\mathrm{MF}}(N)-\frac{1}{2} \operatorname{tr}_{2}\left\{V \operatorname{Ex}\left(\gamma \otimes \gamma-P_{N} \otimes P_{N}\right)\right\}
$$

Proof.

$$
\begin{aligned}
\varepsilon_{\mathrm{HF}}(\gamma)+\frac{1}{2} \operatorname{tr}_{2}\{V E x(\gamma \otimes \gamma)\} & =\operatorname{tr}_{1}\{h \gamma\}+\frac{1}{2} \operatorname{tr}_{2}\{V(\gamma \otimes \gamma)\} \\
& \geqq \operatorname{tr}_{1}\{h \gamma\}+\operatorname{tr}_{2}\left\{V\left(d_{\mathrm{TF}} \otimes \gamma\right)\right\}-\frac{1}{2} \operatorname{tr}_{2}\left\{V\left(d_{\mathrm{TF}} \otimes d_{\mathrm{TF}}\right)\right\} \\
& =\operatorname{tr}_{1}\left\{h_{\mathrm{TF}} \gamma\right\}-\frac{1}{2} \operatorname{tr}_{2}\left\{V\left(d_{\mathrm{TF}} \otimes d_{\mathrm{TF}}\right)\right\} \\
& \geqq \tilde{E}_{\mathrm{MF}}(N)+\frac{1}{2} \operatorname{tr}_{2}\left\{V E x\left(P_{N} \otimes P_{N}\right)\right\}
\end{aligned}
$$

A similar estimate in the opposite direction is as follows.

\section{Lemma 2.}

$$
E_{\mathrm{HF}}(N) \leqq \tilde{E}_{\mathrm{MF}}(N)+\frac{1}{2} D\left(\rho_{N}-\rho_{\mathrm{TF}}, \rho_{N}-\rho_{\mathrm{TF}}\right)
$$

Proof. We estimate

$$
\begin{aligned}
E_{\mathrm{HF}} \leqq & \varepsilon_{\mathrm{HF}}\left(P_{N}\right) \\
= & \operatorname{tr}_{1}\left\{h P_{N}\right\}+\frac{1}{2} \operatorname{tr}_{2}\left\{V(1-E x)\left(P_{N} \otimes P_{N}\right)\right\} \\
\leqq & \operatorname{tr}_{1}\left\{h_{\mathrm{TF}} P_{N}\right\}+\frac{1}{2} \operatorname{tr}_{2}\left\{V\left[\left(P_{N}-d_{\mathrm{TF}}\right) \otimes\left(P_{N}-d_{\mathrm{TF}}\right)\right]\right\} \\
& \quad-\frac{1}{2} \operatorname{tr}_{2}\left\{V \operatorname{Ex}\left(P_{N} \otimes P_{N}\right)\right\} .
\end{aligned}
$$

Here enters $\operatorname{tr}_{1}\left\{P_{N}\right\} \leqq N$.

\section{Exchange Estimates}

From Lemmas 1 and 2 we see that the error bound in Theorem 1 essentially asserts the smallness of

$$
\frac{1}{2} \operatorname{tr}_{2}\left\{V \operatorname{Ex}\left(\gamma \otimes \gamma-P_{N} \otimes P_{N}\right)\right\}
$$


provided $\varepsilon_{\mathrm{HF}}(\gamma)-E_{\mathrm{HF}}(N)$ is small enough, and

$$
\frac{1}{2} \operatorname{tr}_{2}\left\{V \operatorname{Ex}\left(P_{N} \otimes P_{N}\right)\right\}=c_{D} \int \rho_{\mathrm{TF}}^{4 / 3}(x) d^{3} x .
$$

We estimate the above quantities by means of the following lemma.

Lemma 3. Let $0 \leqq a, b \leqq 1, \operatorname{tr}_{1}\{a\}, \operatorname{tr}_{1}\{b\}<\infty$ and $X=X^{2}$ be bounded selfadjoint operators on $\mathscr{H}$. Then

$$
\begin{aligned}
& \left|\operatorname{tr}_{2}\{(X \otimes X) \operatorname{Ex}(a \otimes a-b \otimes b)\}\right| \\
& \quad \leqq\left(\operatorname{tr}_{1}\left\{X(a-b)^{2}\right\}\right)^{1 / 2} \cdot \min \left\{\left(2 \operatorname{tr}_{1}\{X(a+b)\}\right)^{1 / 2}, \operatorname{tr}_{1}\{X(a+b)\}\right\} .
\end{aligned}
$$

Proof. Let $E, F$ be trace class and $\left\{\varphi_{i}\right\}_{l \in \mathbb{N}}$ an ON-basis in $\mathscr{H}$. Then

$$
\begin{aligned}
\operatorname{tr}_{2}\{E x(E \otimes F)\} & =\sum_{i, j=1}^{\infty}\left\langle\varphi_{i} \otimes \varphi_{j} \mid(E \otimes F) \varphi_{j} \otimes \varphi_{i}\right\rangle \\
& =\sum_{i, j=1}^{\infty}\left\langle\varphi_{i}|E| \varphi_{j}\right\rangle\left\langle\varphi_{j}|F| \varphi_{i}\right\rangle=\operatorname{tr}_{1}\{E F\}
\end{aligned}
$$

Hence,

$$
\begin{aligned}
\left|\operatorname{tr}_{2}\{(X \otimes X) E x(a \otimes a-b \otimes b)\}\right| & =\left|\operatorname{tr}_{1}\{a X a X-b X b X\}\right| \\
& =\left|\operatorname{tr}_{1}\{(a-b) X(a+b) X\}\right| \\
& =\left|\operatorname{tr}_{1}\{(a-b) X[X(a+b) X]\}\right| \\
& \leqq\left(\operatorname{tr}_{1}\left\{X(a-b)^{2}\right\}\right)^{1 / 2}\left(\operatorname{tr}_{1}\left\{[X(a+b) X]^{2}\right\}\right)^{1 / 2}
\end{aligned}
$$

Observe that $0 \leqq X F X \leqq 2$ implies

$$
\operatorname{tr}_{1}\left\{[X F X]^{2}\right\} \leqq 2 \operatorname{tr}_{1}\{X F X\}=2 \operatorname{tr}_{1}\{X F\}
$$

On the other hand,

$$
\operatorname{tr}_{1}\left\{[X F X]^{2}\right\} \leqq\left(\operatorname{tr}_{1}\{X F X\}\right)^{2},
$$

because of $0 \leqq X F X$. This, inserted in (28) proves the assertion.

Patterned after Lemma 5 in [1], we can exploit Lemma 3 to show

Lemma 4. Let $0 \leqq a, b \leqq 1, \operatorname{tr}_{1}\{a\}, \operatorname{tr}_{1}\{b\}<\infty$ be bounded self-adjoint operators on $\mathscr{H}$. Then, for any $\varepsilon>0$,

$$
\begin{aligned}
& \operatorname{tr}_{2}\{(V \operatorname{Ex}(a \otimes a-b \otimes b)\} \mid \\
& \quad \leqq C_{\varepsilon}\left(\frac{\operatorname{tr}_{1}\left\{(a-b)^{2}\right\}}{\operatorname{tr}_{1}\{a+b\}}\right)^{1 / 2-\varepsilon}\|\rho[a+b]\|_{1}^{1 / 2}\|\rho[a+b]\|_{5 / 3}^{5 / 6},
\end{aligned}
$$

where $C_{\varepsilon}:=\frac{1152(100 \pi)^{1 / 3}}{3 \pi 6^{1 / 3}} \varepsilon^{-1 / 2}$.

Proof. Using $X_{(r, z)}=X_{(r, z)}^{\dagger}=X_{(r, z)}^{2}:=\chi_{\{x|| x-z \mid \leqq r\}} \otimes 1(\sigma)$, we may decompose (see [3])

$$
V=\frac{1}{\pi} \int d^{3} z \int_{0}^{\infty} \frac{d r}{r^{5}}\left(X_{(r, z)} \otimes X_{(r, z)}\right)
$$


An application of Lemma 3 then yields

$$
\begin{aligned}
\mid \operatorname{tr}_{2}\{ & V E x(a \otimes a-b \otimes b)\} \mid \\
\leqq & \frac{1}{\pi} \int d^{3} z \int_{0}^{\infty} \frac{d r}{r^{5}}\left|\operatorname{tr}_{2}\left\{\left(X_{(r, z)} \otimes X_{(r, z)}\right) \operatorname{Ex}(a \otimes a-b \otimes b)\right\}\right| \\
\leqq & \frac{1}{\pi} \int d^{3} z \int_{0}^{\infty} \frac{d r}{r^{5}}\left(\operatorname{tr}_{1}\left\{X_{(r, z)}(a-b)^{2}\right\}\right)^{1 / 2} \\
& \quad \times \min \left\{\left(2 \operatorname{tr}_{1}\left\{X_{(r, z)}(a+b)\right\}\right)^{1 / 2}, \operatorname{tr}_{1}\left\{X_{(r, z)}(a+b)\right\}\right\} .
\end{aligned}
$$

Observe that $\operatorname{tr}_{1}\left\{X_{(r, z)} d\right\}=\int_{|x-z| \leqq r} \rho[d](x) d^{3} x$. Denoting $\rho_{-}:=\rho\left[(a-b)^{2}\right]$ and $\rho_{+}:=\rho[a+b]$, the integrand on the right hand of (33) is bounded above by

$$
\begin{aligned}
& \frac{1}{\pi} \int d^{3} z\left\{\int_{0}^{R(z)} \frac{d r}{r^{5}}\left(\int_{|x-z| \leqq r} \rho_{-}(x) d^{3} x\right)^{1 / 2}\left(\int_{|x-z| \leqq r} \rho_{+}(x) d^{3} x\right)\right. \\
& \left.\quad+2 \int_{R(z)}^{\infty} \frac{d r}{r^{5}}\left(\int_{|x-z| \leqq r} \rho_{-}(x) d^{3} x\right)^{1 / 2}\left(\int_{|x-z| \leqq r} \rho_{+}(x) d^{3} x\right)^{1 / 2}\right\},
\end{aligned}
$$

for any measurable choice of $R(z)$. We introduce the maximal function for $\rho \in L^{1}\left(\mathbb{R}^{3}\right)$ by

$$
M[\rho](z):=\sup _{r>0}\left(\frac{4 \pi r^{3}}{3}\right)^{-1} \int_{|x-z| \leqq r} \rho(x) d^{3} x .
$$

With the aid of the maximal functions $M_{-}:=M\left[\rho_{-}\right]$and $M_{+}:=M\left[\rho_{+}\right]$, choosing $R(z):=4\left(4 \pi M_{+}(z) / 3\right)^{-1 / 3}$, we obtain the following upper bound on (34):

$$
\begin{aligned}
\left(\frac{4 \pi}{3}\right) & \int d^{3} z M_{-}^{1 / 2}(z) M_{+}^{1 / 2}(z)\left[\left(\frac{4 \pi}{3}\right)^{1 / 2} M_{+}^{1 / 2}(z) \int_{0}^{R(z)} \frac{d r}{r^{1 / 2}}+2 \int_{R(z)}^{\infty} \frac{d r}{r^{2}}\right] \\
& \leqq 3\left(\frac{4 \pi}{3}\right)^{4 / 3} \int d^{3} z M_{-}^{1 / 2}(z) M_{+}^{5 / 6}(z) \\
& \leqq \frac{48}{\pi}\left(\frac{25}{2}\right)^{1 / 3} \varepsilon^{-1 / 2}\left(\int \rho_{-}(x) d^{3} x\right)^{1 / 2-\varepsilon}\left\|\rho_{+}\right\|_{1}^{\varepsilon} \cdot\left\|\rho_{+}\right\| \|_{5 / 3}^{5 / 6} .
\end{aligned}
$$

To get the last inequality, we applied successively the Hölder-, the maximal- and again the Hölder inequality and assumed $0<\varepsilon<1 / 6$. This is very similar to (88)-(91) in [1].

The form of Lemma 4 is a little inconvenient and for the cases of interest may easily be reduced to

Lemma 5. Let $0 \leqq a, b \leqq 1$ be two self-adjoint operators on $\mathscr{H}$ with $\operatorname{tr}_{1}\{b\} \leqq \operatorname{tr}_{1}\{a\}=N<\infty$ and $\operatorname{tr}_{1}\{h a\}, \operatorname{tr}_{1}\{h b\}<0$. Then there exists $C \geqq 0$ such that for all $0<\varepsilon<1 / 6$,

$$
\left|\operatorname{tr}_{2}\{V \operatorname{Ex}(a \otimes a-b \otimes b)\}\right| \leqq \frac{C}{\varepsilon^{1 / 2}}\left(\frac{\operatorname{tr}_{1}\{a(1-b)\}}{N}\right)^{1 / 2-\varepsilon} N^{2 / 3} Z
$$

Proof. Using a kinetic energy bound of Lieb [10] (see also [1]), we derive $\|\rho[f]\|_{5 / 3}^{5 / 3} \leqq c Z^{2} \operatorname{tr}_{1}\{f\}$ from $0 \leqq f \leqq 1$ and $\operatorname{tr}_{1}\{h f\} \leqq 0$. We insert this, 


$$
\begin{aligned}
& (x+y)^{p} \leqq 2^{p}\left(x^{p}+y^{p}\right) \text { for } x, y \geqq 0, \text { and } \\
& \operatorname{tr}_{1}\left\{(a-b)^{2}\right\}=\operatorname{tr}_{1}\left\{a^{2}+b^{2}-2 a b\right\} \leqq 2 N-2 \operatorname{tr}\{a b\}=2 \operatorname{tr}_{1}\{a(1-b)\}
\end{aligned}
$$

into Lemma 4 and arrive at the upper bound in (37). The lower bound is similar.

\section{Bounds on Truncated Particle Numbers}

In the preceding section we estimated the difference of exchange terms induced by 1 -pdm, $a, b$ in terms of a truncated particle number $\operatorname{tr}_{1}\{a(1-b)\}$. For large molecules we bound these quantities in the semiclassical limit. To this end we use a coherent state method similar to Lieb [11].

For a radial, normalized $g \in L^{2}\left(\mathbb{R}^{3}\right)$ we define $f_{p q}(z):=g(z-q) \exp (i p z)$. $f_{p q} \in L^{2}\left(\mathbb{R}^{3}\right)$ is normalized and we denote $f_{p q}^{\sigma}:=f_{p q} \otimes \delta_{\sigma, \cdot} \in \mathscr{H}$, being normalized, as well. It is easy to see $[11,8]$ that weakly in $H^{2}\left(\mathbb{R}^{3}\right) \otimes \mathbb{C}^{m}$ and for $\phi \in L^{5 / 2}\left(\mathbb{R}^{3}\right)+L^{\infty}\left(\mathbb{R}^{3}\right)$ hold

$$
\begin{gathered}
\sum_{\sigma=1}^{m} \int \frac{d^{3} p d^{3} q}{(2 \pi)^{3}}\left|f_{p q}^{\sigma}\right\rangle\left\langle f_{p q}^{\sigma}\left|=1, \quad \sum_{\sigma=1}^{m} \int \frac{d^{3} p d^{3} q}{(2 \pi)^{3}} p^{3}\right| f_{p q}^{\sigma}\right\rangle\left\langle f_{p q}^{\sigma}\right|=-\Delta+\|\nabla g\|_{2}^{2}, \\
\sum_{\sigma=1}^{m} \int \frac{d^{3} p d^{3} q}{(2 \pi)^{3}} \phi(q)\left|f_{p q}^{\sigma}\right\rangle\left\langle\left. f_{p q}^{\sigma}|=\phi *| g\right|^{2},\right. \\
\left\langle f_{p q}^{\sigma}|-\Delta| f_{p q}^{\sigma}\right\rangle=p^{2}+\|\nabla g\|_{2}^{2}, \quad\left\langle f_{p q}^{\sigma} \mid \phi f_{p q}^{\sigma}\right\rangle=\left[\phi *|g|^{2}\right](q) \\
\left\langle f_{p q}^{\sigma} \otimes f_{\tilde{p} \tilde{q}}^{\tilde{\sigma}} \mid V\left(f_{p q}^{\sigma} \otimes f_{\tilde{p} \tilde{q}}^{\tilde{\sigma}}\right)\right\rangle \leqq \frac{1}{|q-\tilde{q}|}
\end{gathered}
$$

We choose $g(x):=g_{\lambda}(x)=\lambda^{-3 / 2} g_{1}(x / \lambda), g_{1}(x):=\pi^{-3 / 4} \exp \left(-x^{2} / 2\right)$ and define a bounded operator $0 \leqq d_{\lambda} \leqq 1$ on $\mathscr{H}$ by

$$
d_{\lambda}:=\sum_{\sigma=1}^{m} \int \frac{d^{3} p d^{3} q}{(2 \pi)^{3}} M(p, q)\left|f_{p q}^{\sigma}\right\rangle\left\langle f_{p q}^{\sigma}\right|,
$$

where $M(p, q):=\Theta\left[\mu-h_{\mathrm{TF}}(p, q)\right], h_{\mathrm{TF}}(p, q):=p^{2}-\phi_{\mathrm{TF}}$. Note that, via the TFequation [11],

$$
\left[\phi_{\mathrm{TF}}(q)-\mu\right]_{+}=\left(\frac{6 \pi^{2}}{m}\right)^{2 / 3} \rho_{\mathrm{TF}}^{2 / 3}(q)
$$

away from the nuclei. Then

$$
\sum_{\sigma=1}^{m} \int \frac{d^{3} p}{(2 \pi)^{3}} M(p, q)=\rho_{\mathrm{TF}}(q)
$$

and, thus, $\operatorname{tr}_{1}\left\{d_{\lambda}\right\}=\left\|\rho_{\mathrm{TF}}\right\|_{1}=\min \{N, Z\}$. In what follows we will frequently use

$$
\begin{aligned}
0 & \leqq \int d^{3} q\left\{\phi_{\mathrm{TF}}(q)-\left[\phi_{\mathrm{TF}} *\left|g_{\lambda}\right|^{2}\right](q)\right\} \rho(q) \\
& \leqq \int d^{3} q \sum_{j=1}^{K}\left\{\frac{Z_{j}}{\left|q-R_{j}\right|}-\int d^{3} x \frac{Z_{j}\left|g_{\lambda}(x-q)\right|^{2}}{\left|x-R_{j}\right|}\right\} \rho(q) \\
& \leqq c Z \lambda^{1 / 5}\|\rho\|_{5 / 3}
\end{aligned}
$$


for nonnegative $\rho \in L^{5 / 3}\left(\mathbb{R}^{3}\right) \cap L^{1}\left(\mathbb{R}^{3}\right)$. The first two inequalities in (44) follow from a subharmonicity argument (see [11]) and the third from the Hölder- and Jensen inequality and the scaling properties of $g_{\lambda}$. Now, we prove the following lemma

Lemma 6. Let $0 \leqq \gamma \leqq 1, \operatorname{tr}_{1}\{h \gamma\} \leqq 0$ be either

(i) the 1-pdm $\gamma=\gamma_{\psi}$ of a $Z^{5 / 3}$-approximate ground state $\psi_{N} \in \mathscr{H}_{N}$, i.e. $\left\langle\psi_{N} \mid H_{N} \psi_{N}\right\rangle-E_{Q}(N) \leqq Z^{5 / 3}$, or

(ii) $\gamma=\gamma_{\phi}$ for a Slater determinant $\phi_{N} \in S D_{N}$ and $\varepsilon_{\mathrm{HF}}(\gamma)-E_{\mathrm{HF}}(N) \leqq Z^{5 / 3}$, or

(iii) $\gamma=P_{N}$.

Then

$$
\operatorname{tr}_{1}\left\{h_{\mathrm{TF}} \gamma\right\} \leqq m \int \frac{d^{3} p d^{3} q}{(2 \pi)^{3}} h_{\mathrm{TF}}(p, q) M(p, q)+c\left(Z^{5 / 3}+Z \lambda^{-2}+Z^{12 / 5} \lambda^{1 / 5}\right) .
$$

Proof. Both in case (i) and (ii) we apply the Lieb-Oxford inequality [13]

$$
\left\langle\psi_{N} \mid\left(\sum_{i \neq j} \frac{1}{\left|x_{i}-x_{j}\right|}\right) \psi_{N}\right\rangle \geqq D\left(\rho\left[\gamma_{\psi}\right], \rho\left[\gamma_{\psi}\right]\right)-1.68 \int d^{3} x \rho\left[\gamma_{\psi}\right]^{4 / 3}(x) .
$$

This gives us

$$
\begin{aligned}
\operatorname{tr}_{1}\left\{h_{\mathrm{TF}} \gamma_{\psi}\right\} \leqq & \operatorname{tr}_{1}\left\{h \gamma_{\psi}\right\}+\frac{1}{2} D\left(\rho\left[\gamma_{\psi}\right], \rho\left[\gamma_{\psi}\right]\right)+\frac{1}{2} D\left(\rho_{\mathrm{TF}}, \rho_{\mathrm{TF}}\right) \\
\leqq & \left\langle\psi_{N} \mid H_{N} \psi_{N}\right\rangle+\frac{1}{2} D\left(\rho_{\mathrm{TF}}, \rho_{\mathrm{TF}}\right) \\
& +c\left\|\rho\left[\gamma_{\psi}\right]\right\|_{1}^{1 / 2}\left\|\rho\left[\gamma_{\psi}\right]\right\|_{5 / 3}^{5 / 6} .
\end{aligned}
$$

For both (i) and (ii) $\operatorname{tr}_{1}\{h \gamma\} \leqq 0$ implies $\|\rho[\gamma]\|_{5 / 3}^{5 / 3} \leqq c Z^{7 / 3}$ (see $[11,1]$ ). This yields

$$
\operatorname{tr}_{1}\left\{h_{\mathrm{TF}} \gamma_{\psi}\right\} \leqq E_{\mathrm{HF}}(N)+\frac{1}{2} D\left(\rho_{\mathrm{TF}}, \rho_{\mathrm{TF}}\right)+c Z^{5 / 3} .
$$

Now, by (40) and (44)

$$
\begin{aligned}
E_{\mathrm{HF}}(N) & +\frac{1}{2} D\left(\rho_{\mathrm{TF}}, \rho_{\mathrm{TF}}\right) \\
\leqq & \operatorname{tr}_{1}\left\{h d_{\lambda}\right\}+\frac{1}{2} \operatorname{tr}_{2}\left\{V\left(d_{\lambda} \otimes d_{\lambda}\right)\right\}+\frac{1}{2} D\left(\rho_{\mathrm{TF}}, \rho_{\mathrm{TF}}\right) \\
\leqq & m \int \frac{d^{3} p d^{3} q}{(2 \pi)^{3}} M(p, q)\left(p^{2}+\left\|\nabla g_{\lambda}\right\|_{2}^{2}-\sum_{j=1}^{K} \int d^{3} x \frac{Z_{j}\left|g_{\lambda}(x-q)\right|^{2}}{\left|x-R_{j}\right|}\right) \\
& \quad+D\left(\rho_{\mathrm{TF}}, \rho_{\mathrm{TF}}\right) \\
\leqq & m \int \frac{d^{3} p d^{3} q}{(2 \pi)^{3}} M(p, q)\left(p^{2}-\phi_{\mathrm{TF}}(q)\right)+c Z \lambda^{1 / 5}\left\|\rho_{\mathrm{TF}}\right\|_{5 / 3} \\
& \quad+N\left\|\nabla g_{\lambda}\right\|_{2}^{2} .
\end{aligned}
$$


Inserting $\left\|\rho_{\mathrm{TF}}\right\|_{5 / 3} \leqq c Z^{7 / 5}$ and $\left\|\nabla g_{\lambda}\right\|_{2}^{2} \leqq c \lambda^{-2}$ gives (45) in Case (i) and (ii). In Case (iii) we observe

$$
\begin{aligned}
\operatorname{tr}_{1}\left\{h_{\mathrm{TF}} P_{N}\right\} \leqq & \operatorname{tr}_{1}\left\{h_{\mathrm{TF}} d_{\lambda}\right\} \\
\leqq & m \int \frac{d^{3} p d^{3} q}{(2 \pi)^{3}} M(p, q)\left(p^{2}+\left\|\nabla g_{\lambda}\right\|_{2}^{2}-\left[\phi_{\mathrm{TF}} *\left|g_{\lambda}\right|^{2}\right](q)\right) \\
\leqq & m \int \frac{d^{3} p d^{3} q}{(2 \pi)^{3}} M(p, q)\left(p^{2}-\phi_{\mathrm{TF}}(q)\right) \\
& +c Z^{12 / 5} \lambda^{1 / 5}+c Z \lambda^{-2},
\end{aligned}
$$

using (44) again.

We define $F(\lambda):=Z^{5 / 3}+Z \lambda^{-2}+Z^{12 / 5} \lambda^{1 / 5}$. Now we derive a bound on $\operatorname{tr}_{1}\left\{d_{\lambda}(1-\gamma)\right\}$ in all the three above cases by $(45)$.

Lemma 7. Let $0 \leqq \gamma \leqq 1, \operatorname{tr}_{1}\{h \gamma\} \leqq 0, \operatorname{tr}_{1}\{\gamma\} \leqq N$ fulfill

$$
\operatorname{tr}_{1}\left\{h_{\mathrm{TF}} \gamma\right\} \leqq m \int \frac{d^{3} p d^{3} q}{(2 \pi)^{3}} h_{\mathrm{TF}}(p, q) M(p, q)+c F(\lambda) .
$$

Then

$$
\operatorname{tr}_{1}\left\{d_{\lambda}(1-\gamma)\right\} \leqq c K F^{3 / 7}(\lambda)
$$

Proof. For any $E>0$ we have

$$
\begin{aligned}
\operatorname{tr}_{1}\left\{d_{\lambda}(1-\gamma)\right\}= & \sum_{\sigma=1}^{m} \int \frac{d^{3} p d^{3} q}{(2 \pi)^{3}} M(p, q)\left\langle f_{p q}^{\sigma} \mid(1-\gamma) f_{p q}^{\sigma}\right\rangle \\
\leqq & m \int_{-\mu \geqq h_{\mathrm{TF}}(p, q) \geqq-\mu-E} \frac{d^{3} p d^{3} q}{(2 \pi)^{3}} \\
& +\frac{1}{E} \sum_{\sigma=1}^{m} \int \frac{d^{3} p d^{3} q}{(2 \pi)^{3}} M(p, q)\left[-h_{\mathrm{TF}}(p, q)\right]\left\langle f_{p q}^{\sigma} \mid(1-\gamma) f_{p q}^{\sigma}\right\rangle \\
\leqq & m \quad-\mu \geqq h_{\mathrm{TF}}(p, q) \geqq-\mu-E \frac{d^{3} p d^{3} q}{(2 \pi)^{3}} \\
& +\frac{1}{E}\left\{\operatorname{tr}_{1}\left\{h_{\mathrm{TF}} \gamma\right\}-m \int \frac{d^{3} p d^{3} q}{(2 \pi)^{3}} M(p, q) h_{\mathrm{TF}}(p, q)\right. \\
& \left.+N\left\|\nabla g_{\lambda}\right\|_{2}^{2}+\int d^{3} q\left(\phi_{\mathrm{TF}}(q)-\left[\phi_{\mathrm{TF}} *\left|g_{\lambda}\right|^{2}\right](q)\right) \rho[\gamma](q)\right\} .
\end{aligned}
$$

By (44), $\left\|\nabla g_{\lambda}\right\|_{2}^{2} \leqq c \lambda^{-2},\|\rho[\gamma]\|_{5 / 3}^{5 / 3} \leqq c Z^{7 / 3}$ and (51), this gives

$$
\operatorname{tr}_{1}\left\{d_{\lambda}(1-\gamma)\right\} \leqq m \int_{-\mu \geqq h_{\mathrm{TF}}(p, q) \geqq-\mu-E} \frac{d^{3} p d^{3} q}{(2 \pi)^{3}}+\frac{c}{E} F(\lambda) .
$$

We distinguish $N \geqq Z$ from $N<Z$. In the former case $\mu=0$ and it was shown in [1] that

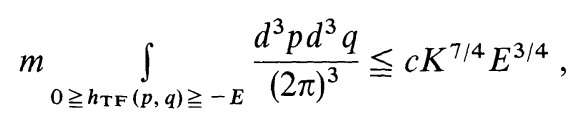


which, choosing $E:=K^{-1} F^{4 / 7}(\lambda)$, leads us to (52). Actually, (55) required $E=o\left(Z^{4 / 3}\right)$, but if $E \geqq c Z^{4 / 3}$ then (55) is trivial anyway.

If $N<Z$ we emphasize $\phi_{\mathrm{TF}, N}(q):=\phi_{\mathrm{TF}}(q), \mu_{N}:=\mu$ and $\rho_{\mathrm{TF}, N}(q):=\rho_{\mathrm{TF}}(q)$. From TF-theory follows $[11]\left[\phi_{\mathrm{TF}, N}(q)-\mu_{N}\right]_{+}=\alpha \rho_{\mathrm{TF}, N}^{2 / 3}(q) \leqq \alpha \rho_{\mathrm{TF}, Z}^{2 / 3}(q)=\phi_{\mathrm{TF}, Z}(q)$, with $\alpha:=\left(6 \pi^{2} m^{-1}\right)^{2 / 3}$. Thus

$$
\begin{aligned}
m & \int_{-\mu \geqq h_{\mathrm{TF}}(p, q) \geqq-\mu-E} \frac{d^{3} p d^{3} q}{(2 \pi)^{3}} \\
& =\frac{m}{6 \pi^{2}} \int_{\phi_{\mathrm{TF}}, N \geqq \mu_{N}} d^{3} q\left\{\left[\phi_{\mathrm{TF}, N}(q)-\mu_{N}\right]_{+}^{3 / 2}-\left[\left(\phi_{\mathrm{TF}, N}(q)-\mu_{N}\right)_{+}-E\right]_{+}^{3 / 2}\right\} \\
& \leqq \frac{m}{6 \pi^{2}} \int d^{3} q\left\{\phi_{\mathrm{TF}, Z}(q)^{3 / 2}-\left[\phi_{\mathrm{TF}, Z}(q)-E\right]_{+}^{3 / 2}\right\} \\
& \leqq c K^{7 / 4} E^{3 / 4},
\end{aligned}
$$

again, by (55).

\section{The Dirac Exchange Correction}

In this section we link the exchange term induced by $d_{\lambda}$ with the Dirac exchange correction $c_{D}\left\|\rho_{\mathrm{TF}}\right\|_{4 / 3}^{4 / 3}$ (see $[2,14]$ ). Recall from the last section we may represent the 1-pdm $d_{\lambda}$ by its integral kernel

$$
d_{\lambda}(x, \mu \mid y, v):=\int \frac{d^{3} p d^{3} q}{(2 \pi)^{3}} M(p, q) g_{\lambda}(x-q) g_{\lambda}(y-q) e^{i p(x-y)} \delta_{\mu v} .
$$

The exchange term induced by $d_{\lambda}$ reads

$$
W_{e x}:=\operatorname{tr}_{2}\left\{V \operatorname{Ex}\left(d_{\lambda} \otimes d_{\lambda}\right)\right\}=\frac{m}{2} \int \frac{d^{3} x d^{3} y}{|x-y|}\left|d_{\lambda}(x \mid y)\right|^{2},
$$

where $d_{\lambda}(x \mid y):=d_{\lambda}(x, \mu \mid y, \mu)$. Our specific choice of $g_{\lambda}$ allows us to compute the right hand of (58) almost explicitly. It requires a tedious integration and the result is

\section{Lemma 8.}

$$
\begin{aligned}
& \left|\frac{1}{2} \operatorname{tr}_{2}\left\{V \operatorname{Ex}\left(d_{\lambda} \otimes d_{\lambda}\right)\right\}-c_{D} \int d^{3} x \rho_{\mathrm{TF}}^{4 / 3}(x)\right| \\
& \quad \leqq c_{v} K^{2}\left[Z \lambda^{-1}+Z^{2} \ln (Z)\left(\lambda+Z^{-1 / 3-v}\right)\right]
\end{aligned}
$$

for any $v>0$.

Proof. We start with a change of variables $2 s:=x+y, 2 r:=x-y$. Moreover, we observe $g_{\lambda}(z+r) g_{\lambda}(z-r)=g_{\lambda}^{2}(z) \cdot \exp \left(-r^{2} / \lambda^{2}\right)$. Hence, abbreviating $p_{f}(q):=$ $\left[\phi_{\mathrm{TF}}(q)-\mu\right]_{+}^{1 / 2}=\left(6 \pi^{2} m^{-1}\right)^{1 / 3} \rho_{\mathrm{TF}}^{1 / 3}(q)$,

$$
\begin{aligned}
W_{e x}= & 2 m \int \frac{d^{3} s d^{3} r}{|r|} e^{-r^{2} / \lambda^{2}}\left|\int \frac{d^{3} p d^{3} q}{(2 \pi)^{3}} M(p, s+q) g_{\lambda}^{2}(q) e^{2 i \tilde{p} \cdot \vec{r}}\right|^{2} \\
= & 2 m \int d^{3} s \int \frac{d^{3} q d^{3} \tilde{q}}{(2 \pi)^{6}} g_{\lambda}^{2}(q) g_{\lambda}^{2}(\tilde{q}) \int_{0}^{p_{f}(s+q)} p^{2} d p \int_{0}^{p_{f}(s+\tilde{q})} \tilde{p}^{2} d \tilde{p} \\
& \times \int_{0}^{\infty} r e^{-r^{2} / \lambda^{2}} d r \int d \Omega_{p} d \Omega_{\tilde{p}} 2 \pi \int_{-1}^{1} d x \exp (2 i|\vec{p}-\overrightarrow{\tilde{p}}| r x) .
\end{aligned}
$$


Here, we wrote $\vec{x}$ whenever necessary to distinguish it from $|\vec{x}|$. We observe

$$
\int d \Omega_{p} d \Omega_{\tilde{p}} 2 \pi \int_{-1}^{1} d x \exp (2 i|\vec{p}-\overrightarrow{\tilde{p}}| r x)=\frac{2(2 \pi)^{3}}{p \tilde{p} r^{2}} \sin (2 r p) \sin (2 r \tilde{p}) .
$$

This yields

$$
W_{e x}=\frac{4 m}{(2 \pi)^{3}} \int d^{3} s \int_{0}^{\infty} d r \frac{e^{-r^{2} / \lambda^{2}}}{r}\left(\int d^{3} q g_{\lambda}^{2}(q) \int_{0}^{p_{f}(s+q)} p \sin (2 r p) d p\right)^{2} .
$$

Defining

$$
\begin{aligned}
\varepsilon_{1}:= & \frac{4 m}{(2 \pi)^{3}} \int d^{3} s \int_{0}^{\infty} d r \frac{e^{-r^{2} / \lambda^{2}}}{r} \int d^{3} q g_{\lambda}^{2}(q) d^{3} \tilde{q} g_{\lambda}^{2}(\tilde{q}) \\
& \left(\int_{0}^{p_{f}(s+q)} p \sin (2 r p) d p-\int_{0}^{p_{f}(s+\tilde{q})} p \sin (2 r p) d p\right)^{2}
\end{aligned}
$$

and

$$
\varepsilon_{2}:=\frac{4 m}{(2 \pi)^{3}} \int d^{3} s p_{f}^{4 / 3}(s) \int_{0}^{\infty} \frac{[\sin (r)-r \cos (r)]^{2} d r}{r^{5}}\left[1-\exp \left(-\frac{r^{2}}{2 p_{f}^{2}(s) \lambda^{2}}\right)\right],
$$

we can extract the leading part from $W_{e x}$;

$$
\begin{aligned}
W_{e x} & =\frac{4 m}{(2 \pi)^{3}}\left(\int_{0}^{\infty} \frac{[\sin (r)-r \cos (r)]^{2}}{r^{5}} d r\right) \int p_{f}^{4}(s) d^{3} s-\varepsilon_{1}-\varepsilon_{2} \\
& =c_{D} \int \rho_{\mathrm{TF}}^{4 / 3}(x) d^{3} x-\varepsilon_{1}-\varepsilon_{2} .
\end{aligned}
$$

It remains to estimate $\varepsilon_{1}, \varepsilon_{2} \geqq 0$. We start with $\varepsilon_{2}$. Using $1-e^{-\alpha^{2}} \leqq \alpha^{2} e^{-\alpha^{2}}$, we get for $f(x) \geqq 0$,

$$
\begin{aligned}
\int_{0}^{\infty} f(x)\left(1-e^{-x^{2} / a^{2}}\right) d x & \leqq \frac{1}{a^{2}}\left(\int_{0}^{\infty} \frac{f(x) d x}{x}\right)^{1 / 2}\left(\int_{0}^{\infty} x e^{-x^{2} / a^{2}} d x\right)^{1 / 2} \\
& \leqq \frac{c}{a}\left(\int_{0}^{\infty} \frac{f(x) d x}{x}\right)^{1 / 2} .
\end{aligned}
$$

This, inserted in (64), yields

$$
\varepsilon_{2} \leqq \frac{c}{\lambda} \int p_{f}^{3}(s) d^{3} s \leqq \frac{c Z}{\lambda}
$$

The estimate on $\varepsilon_{1}$ is more delicate. We use

$$
\left(\int_{2 r p_{f}}^{2 r \tilde{p}_{f}} p \sin (p) d p\right)^{2} \leqq \frac{64 r^{6}}{9}\left(p_{f}^{3}-\tilde{p}_{f}^{3}\right)^{2}
$$

for small $r>0$ and

$$
\left[\sin \left(2 r p_{f}\right)-2 r p_{f} \cos \left(2 r p_{f}\right)-\sin \left(2 r \tilde{p}_{f}\right)+2 r \tilde{p}_{f} \cos \left(2 r \tilde{p}_{f}\right)\right]^{2} \leqq c r^{2}\left[p_{f}+\tilde{p}_{f}\right]^{2}
$$

for large $r>0$, denoting $p_{f}:=p_{f}(s+q), \tilde{p}_{f}:=p_{f}(s+\tilde{q})$. Therefore, for any measurable choice of $R=R(s, q, \tilde{q})$,

$$
\varepsilon_{1} \leqq c \int d^{3} s \int d^{3} q g_{\lambda}^{2}(q) d^{3} \tilde{q} g_{\lambda}^{2}(\tilde{q})\left[\left(p_{f}^{3}-\tilde{p}_{f}^{3}\right) R^{2}+\frac{\left(p_{f}+\tilde{p}_{f}\right)^{2}}{R^{2}}\right] .
$$


We optimize by choosing $R:=\left(p_{f}+\tilde{p}_{f}\right)\left|p_{f}^{3}-\tilde{p}_{f}^{3}\right|^{-1}$. Furthermore, we apply $(a+b)\left|a^{3}-b^{3}\right| \leqq 2\left|a^{4}-b^{4}\right|$ which holds for $a, b \geqq 0$ and obtain

$$
\varepsilon_{1} \leqq c \int d^{3} s \int d^{3} q g_{\lambda}^{2}(q) d^{3} \tilde{q} g_{\lambda}^{2}(\tilde{q})\left|p_{f}^{4}-\tilde{p}_{f}^{4}\right|
$$

We pause to motivate the next step. We would like to estimate

$$
\left|p_{f}^{4}(s+q)-p_{f}^{4}(s+\tilde{q})\right| \leqq \int_{q}^{\tilde{q}}\left|\nabla p_{f}^{4}\left(s+s^{\prime}\right)\right| d s^{\prime},
$$

where $s^{\prime}$ is on the straight line between $q$ and $\tilde{q}$. Then

$$
\begin{aligned}
\varepsilon_{1} & \leqq c \int d^{3} s \int d^{3} q g_{\lambda}^{2}(q) d^{3} \tilde{q} g_{\lambda}^{2}(\tilde{q}) \int_{q}^{\tilde{q}}\left|\nabla p_{f}^{4}\left(s+s^{\prime}\right)\right| d s^{\prime} \\
& \leqq c \int d^{3} q g_{\lambda}^{2}(q) d^{3} \tilde{q} g_{\lambda}^{2}(\tilde{q}) \int_{q}^{\tilde{q}}\left(\int d^{3} s\left|\nabla p_{f}^{4}\left(s+s^{\prime}\right)\right|\right) d s^{\prime} \\
& \leqq c\left(\int\left|\nabla p_{f}^{4}(s)\right| d^{3} s\right) \int d^{3} q g_{\lambda}^{2}(q) d^{3} \tilde{q} g_{\lambda}^{2}(\tilde{q})|q-\tilde{q}| \\
& \leqq c\left\|\nabla p_{f}^{4}\right\|_{1} \cdot \lambda .
\end{aligned}
$$

The trouble with this estimate is that $\left|\nabla p_{f}^{4}\right| \notin L^{1}\left(\mathbb{R}^{3}\right)$, due to singularities at $R_{j}$. To overcome this difficulty we have to cut out the region around the nuclei.

To this end, we introduce a cut-off $p_{0}>0$ :

$$
\begin{aligned}
\left|p_{f}^{4}-\tilde{p}_{f}^{4}\right| \leqq & \Theta\left[p_{0}-p_{f}\right] \Theta\left[p_{0}-\tilde{p}_{f}\right] \cdot\left|p_{f}^{4}-\tilde{p}_{f}^{4}\right|+2 \Theta\left[p_{f}-p_{0}\right] \cdot p_{f}^{4} \\
& +2 \Theta\left[\tilde{p}_{f}-p_{0}\right] \cdot \tilde{p}_{f}^{4} .
\end{aligned}
$$

Hence

$$
\begin{aligned}
\varepsilon_{1} \leqq & c \int d^{3} s \int d^{3} q g_{\lambda}^{2}(q) d^{3} \tilde{q} g_{\lambda}^{2}(\tilde{q}) \Theta\left[p_{0}-p_{f}\right] \Theta\left[p_{0}-\tilde{p}_{f}\right]\left|p_{f}^{4}-\tilde{p}_{f}^{4}\right| \\
& +c \int d^{3} s \int d^{3} q g_{\lambda}^{2}(q) \Theta\left[p_{f}-p_{0}\right] p_{f}^{4} .
\end{aligned}
$$

Now, we estimate

$$
\left|p_{f}^{4}(s+q)-p_{f}^{4}(s+\tilde{q})\right| \leqq \int_{q}^{\tilde{q}}\left|\nabla p_{f}^{4}\left(s+s^{\prime}\right)\right| d s^{\prime},
$$

where we assume the path $s+s^{\prime} \notin A:=\left\{x \in \mathbb{R}^{3} \mid p_{f}(x)>p_{0}\right\}$ and to be of shortest possible length. We choose $p_{0}^{2}:=c Z^{4 / 3+v}$ for some $v>0$ to be picked later. Then $A \subseteq \bigcup_{j=1}^{K} B_{Z^{-1 / 3-v}}\left(R_{j}\right)$, because $p_{f}^{2}(x) \leqq c \sum_{j=1}^{K} Z_{j}\left|x-R_{j}\right|^{-1}$. It follows

$$
\int_{q}^{\tilde{q}} d s^{\prime} \leqq|q-\tilde{q}|+K Z^{-1 / 3-v}
$$

We obtain

$$
\begin{aligned}
& \varepsilon_{1} \leqq c \int d^{3} s \int d^{3} q g_{\lambda}^{2}(q) d^{3} \tilde{q} g_{\lambda}^{2}(\tilde{q}) \int_{q}^{\tilde{q}}\left|\nabla p_{f}^{4}\left(s+s^{\prime}\right)\right| \chi_{\mathbb{R}^{3} \backslash A}\left(s+s^{\prime}\right) d s^{\prime}+c \int_{A} p_{f}^{4}(s) d^{3} s \\
& \leqq c \int d^{3} q g_{\lambda}^{2}(q) d^{3} \tilde{q} g_{\lambda}^{2}(\tilde{q}) \int_{q}^{\tilde{q}}\left(\int d^{3} s\left|\nabla p_{f}^{4}\left(s+s^{\prime}\right)\right| \chi_{\mathbb{R}^{3} \backslash A}\left(s+s^{\prime}\right)\right) d s^{\prime} \\
& \quad+c K Z^{2} \int_{|x| \leqq Z^{-1 / 3-v}} \frac{d^{3} x}{|x|^{2}}
\end{aligned}
$$




$$
\begin{aligned}
& \leqq c\left(\int_{\mathbb{R}^{3} \backslash A}\left|\nabla p_{f}^{4}(s)\right| d^{3} s\right)\left(K Z^{-1 / 3-v}+\int d^{3} q g_{\lambda}^{2}(q) d^{3} \tilde{q} g_{\lambda}^{2}(\tilde{q})|q-\tilde{q}|\right)+c K Z^{5 / 3-v} \\
& \leqq c\left\|\chi_{\mathbb{R}^{3} \backslash A} \nabla p_{f}^{4}\right\|_{1}\left(K Z^{-1 / 3-v}+\lambda\right)+c K Z^{5 / 3-v}
\end{aligned}
$$

We split up the remaining integral by means of $A^{\prime}:=\bigcup_{j=1}^{K} B_{Z^{-1 / 3}}\left(R_{j}\right)$;

$$
\begin{aligned}
\left\|\chi_{\mathbb{R}^{3} \backslash A} \nabla p_{f}^{4}\right\|_{1} & \leqq c K Z^{2} \int_{\left(\mathbb{R}^{3} \backslash A\right) \cap A^{\prime}} \sum_{j=1}^{K} \frac{d^{3} x}{\left|x-R_{j}\right|^{3}}+c \int_{A^{\prime}} Z^{8 / 3}\left|\nabla p_{f, 1}\left(Z^{1 / 3} x\right)\right| d^{3} x \\
& \leqq c_{\nu} K^{2} Z^{2} \ln (Z)+c Z^{2}
\end{aligned}
$$

and we finally arrive at

$$
\varepsilon_{1}+\varepsilon_{2} \leqq c_{v} K^{2}\left[Z \lambda^{-1}+Z^{2} \ln (Z)\left(\lambda+Z^{-1 / 3-v}\right)\right] .
$$

Proof of Theorem 1. The first step of the proof consists in linking the quantum mechanical ground state energy $E_{Q}(N)$ with the Hartree-Fock energy $E_{\mathrm{HF}}(N)$. We invoke Theorem 2:

$$
\left|E_{Q}(N)-E_{\mathrm{HF}}(N)\right| \leqq \frac{c}{\varepsilon^{1 / 3}}\left(\operatorname{tr}_{1}\left\{\gamma_{\psi}-\gamma_{\psi}^{2}\right\}\right)^{1 / 3-\varepsilon} Z^{4 / 3+\varepsilon}
$$

for $0<\varepsilon<1 / 12$ and $\gamma_{\psi}$ being the 1 -pdm of a $Z^{4 / 3}$-approximate ground state $\psi_{N} \in \mathscr{H}_{N}$ of $H_{N}$. From Lemma 1 and 2 we derive

$$
\begin{aligned}
-\frac{1}{2} \operatorname{tr}_{2}\left\{V \operatorname{Ex}\left(\gamma_{\mathrm{HF}} \otimes \gamma_{\mathrm{HF}}-P_{N} \otimes P_{N}\right)\right\}-Z^{4 / 3} \\
\quad \leqq E_{\mathrm{HF}}(N)-\operatorname{tr}_{1}\left\{h_{\mathrm{TF}} P_{N}\right\}+\frac{1}{2} D\left(\rho_{\mathrm{TF}}, \rho_{\mathrm{TF}}\right)-\frac{1}{2} \operatorname{tr}_{2}\left\{V \operatorname{Ex}\left(P_{N} \otimes P_{N}\right)\right\} \\
\quad \leqq \frac{1}{2} D\left(\rho_{N}-\rho_{\mathrm{TF}}, P_{N}-\rho_{\mathrm{TF}}\right)
\end{aligned}
$$

for any orthogonal projection $\gamma_{\mathrm{HF}}=\gamma_{\mathrm{HF}}^{\dagger}=\gamma_{\mathrm{HF}}^{2}, \operatorname{tr}_{1}\left\{\gamma_{\mathrm{HF}}\right\}=N$, with $\varepsilon_{\mathrm{HF}}\left(\gamma_{\mathrm{HF}}\right)-$ $E_{\mathrm{HF}}(N) \leqq Z^{4 / 3}$. Now, we use Lemma 5 combined with the triangle inequality and find for any $\varepsilon \leqq 0$,

$$
\begin{aligned}
& \left|\operatorname{tr}_{2}\left\{V \operatorname{Ex}\left(\gamma_{\mathrm{HF}} \otimes \gamma_{\mathrm{HF}}-P_{N} \otimes P_{N}\right)\right\}\right| \\
& \quad \leqq \frac{c}{\varepsilon^{1 / 2}}\left(\operatorname{tr}_{1}\left\{\gamma_{\mathrm{HF}}\left(1-d_{\lambda}\right)\right\}+\operatorname{tr}_{1}\left\{P_{N}\left(1-d_{\lambda}\right)\right\}\right)^{1 / 2-\varepsilon} Z^{7 / 6+\varepsilon} \\
& \quad \leqq \frac{c}{\varepsilon^{1 / 2}}\left(\operatorname{tr}_{1}\left\{d_{\lambda}\left(1-\gamma_{\mathrm{HF}}\right)\right\}+\operatorname{tr}_{1}\left\{d_{\lambda}\left(1-P_{N}\right)\right\}+Z^{1-2 / 77}\right)^{1 / 2-\varepsilon} Z^{7 / 6+\varepsilon}
\end{aligned}
$$

Note that in the second inequality we used $N \leqq Z+Z^{1-2 / 77}$ and $\operatorname{tr}_{1}\left\{d_{\lambda}\right\}=\min \{N, Z\}$. Of course, this estimate holds for $\operatorname{tr}_{2}\left\{V \operatorname{Ex}\left(d_{\lambda} \otimes d_{\lambda}\right.\right.$ $\left.\left.-P_{N} \otimes P_{N}\right)\right\} \mid$, as well. Moreover, notice that

$$
\operatorname{tr}_{1}\left\{\gamma_{\psi}-\gamma_{\psi}^{2}\right\} \leqq \operatorname{tr}_{1}\left\{d_{\lambda}\left(1-\gamma_{\psi}\right)\right\}+\operatorname{tr}_{1}\left\{\left(1-d_{\lambda}\right) \gamma_{\psi}\right\}=2 \operatorname{tr}_{1}\left\{d_{\lambda}\left(1-\gamma_{\psi}\right)\right\} .
$$


We insert (81), (83) and (84) into (82) and obtain for any $0<\varepsilon<1 / 12$,

$$
\begin{aligned}
\frac{c}{\varepsilon^{1 / 3}}\left(\operatorname{tr}_{1}\left\{d_{\lambda}\left(1-\gamma_{\psi}\right)\right\}\right. & +\operatorname{tr}_{1}\left\{d_{\lambda}\left(1-\gamma_{\mathrm{HF}}\right)\right\} \\
& \left.+\operatorname{tr}_{1}\left\{d_{\lambda}\left(1-P_{N}\right)\right\}+Z^{1-2 / 77}\right)^{1 / 3-\varepsilon} Z^{4 / 3+\varepsilon} \\
\leqq & E_{Q}(N)-\operatorname{tr}_{1}\left\{h_{\mathrm{TF}} P_{N}\right\}+\frac{1}{2} D\left(\rho_{\mathrm{TF}}, \rho_{\mathrm{TF}}\right)-\frac{1}{2} \operatorname{tr}_{2}\left\{V \operatorname{Ex}\left(d_{\lambda} \otimes d_{\lambda}\right)\right\} \\
\leqq & \frac{c}{\varepsilon^{1 / 3}}\left(\operatorname{tr}_{1}\left\{d_{\lambda}\left(1-\gamma_{\psi}\right)\right\}+\operatorname{tr}_{1}\left\{d_{\lambda}\left(1-\gamma_{\mathrm{HF}}\right)\right\}+Z^{1-2 / 77}\right. \\
& +\operatorname{tr}_{1}\left\{d_{\lambda}\left(1-P_{N}\right)\right\}^{1 / 3-\varepsilon} Z^{4 / 3+\varepsilon} \\
& +\frac{1}{2} D\left(\rho_{N}-\rho_{\mathrm{TF}}, \rho_{N}-\rho_{\mathrm{TF}}\right) .
\end{aligned}
$$

In (85) exactly the three cases treated in Lemma 6 occur, which, therefore, fulfill the assumptions in Lemma 7 and imply the bound

$$
\begin{aligned}
& \frac{c}{\varepsilon^{1 / 3}} K^{1 / 3}\left[Z^{7 / 3-2 / 33}+Z \lambda^{2}+Z^{12 / 5} \lambda^{1 / 5}\right]^{1 / 7-\varepsilon} Z^{4 / 3+\varepsilon} \\
& \leqq E_{Q}(N)-\operatorname{tr}_{1}\left\{h_{\mathrm{TF}} P_{N}\right\}+\frac{1}{2} D\left(\rho_{\mathrm{TF}}, \rho_{\mathrm{TF}}\right)-\frac{1}{2} \operatorname{tr}_{2}\left\{V \operatorname{Ex}\left(d_{\lambda} \otimes d_{\lambda}\right)\right\} \\
& \leqq \frac{1}{2} D\left(\rho_{N}-\rho_{\mathrm{TF}}, \rho_{N}-\rho_{\mathrm{TF}}\right) \\
&+\frac{c}{\varepsilon^{1 / 3}} K^{1 / 3}\left[Z^{7 / 3-2 / 33}+Z \lambda^{2}+Z^{12 / 5} \lambda^{1 / 5}\right]^{1 / 7-\varepsilon} Z^{4 / 3+\varepsilon}
\end{aligned}
$$

The error term we pick up when replacing $\frac{1}{2} \operatorname{tr}_{2}\left\{V \operatorname{Ex}\left(d_{\lambda} \otimes d_{\lambda}\right)\right\}$ by $c_{D} \int \rho_{\mathrm{TF}}^{4 / 3}$ is estimated in Lemma 8 and turns out to be small compared to the $\lambda$-dependent error term in (86). More precisely, Theorem 1 follows now from Lemma 8 and the choice $\lambda:=Z^{-21 / 23}$ and $v:=10 / 33$.

Acknowledgement. I would like to thank C. Fefferman for pointing out that the question presently addressed was left open in [1] and H. Siedentop for a remark that simplified the proof of Lemma 3.

\section{References}

1. Bach, V.: Error bound for the Hartree-Fock energy of atoms and molecules. Commun. Math. Phys. 147, 527-549 (1992)

2. Dirac, P.A.M.: Note on exchange phenomena in the Thomas-Fermi atom. Proc. Cambridge Philos. Soc. 26, 376-385 (1931)

3. Fefferman, C.L., de la Llave, R.: Relativistic stability of matter-I. Revista Matematica Iberoamericana 2(1, 2), 119-161 (1986)

4. Fefferman, C.L., Seco, L.A.: An upper bound for the number of electrons in a large ion. Proc. Nat. Acad. Sci. USA 86, 3464-3465 (1989) 
5. Fefferman, C.L., Seco, L.A.: The ground-state energy of a large atom. Bull A.M.S., 1990

6. Fefferman, C.L., Seco, L.A.: The density in a one-dimensional potential. Adv. Math., to appear

7. Helffer, B., Knauf, A., Siedentop, H., Weikard, R.: On the absence of a first order correction for the number of bound states of a Schrödinger operator with Coulomb singularity. Commun. PDE 17, 615-639 (1992)

8. Hunziker, W.: Coherent states. Lecture Notes (unpublished) 1989

9. Ivrii, V.Ja., Sigal, I.M.: Asymptotics of the ground state energies of large Coulomb systems. Annals Math., to appear

10. Lieb, E.H.: The stability of matter. Rev. Mod. Phys. 48, 653-669 (1976)

11. Lieb, E.H.: Thomas-Fermi and related theories of atoms and molecules. Rev. Mod. Phys. 53, 603-604 (1981)

12. Lieb, E.H.: Variational principle for many-fermion systems. Phys. Rev. Lett. 46(7), 457-459 (1981)

13. Lieb, E.H., Oxford, S.: An improved lower bound on the indirect Coulomb energy. Int. J. Quantum Chem. 19, 427-439 (1981)

14. Schwinger, J.: Thomas-Fermi model: The second correction. Phys. Rev. A 24(5), 2353-2361 (1981)

15. Seco, L.A., Sigal, I.M., Solovej, J.-P.: Bound on the ionization energy of large atoms. Commun. Math. Phys. 131, 307-315 (1990)

16. Thirring, W.: Lehrbuch der Mathematischen Physik 4: Quantenmechanik großer Systeme. Berlin, Heidelberg, New York: Springer, 1st ed. 1980

Communicated by B. Simon 